\title{
A light composite scalar in eight-flavor QCD on the lattice
}

\author{
Yasumichi Aoki $^{a}$, Tatsumi Aoyama ${ }^{a}$, Masafumi Kurachi ${ }^{a}$, Toshihide Maskawa ${ }^{a}$, \\ Kohtaroh Miura $^{a}$, Kei-ichi Nagai $^{a}$, Hiroshi Ohki ${ }^{* a}$, Enrico Rinaldi $^{b}$, Akihiro Shibata $^{c}$, \\ Koichi Yamawaki ${ }^{a}$, and Takeshi Yamazaki ${ }^{a}$
}

\section{(LatKMI Collaboration)}

${ }^{a}$ Kobayashi-Maskawa Institute for the Origin of Particles and the Universe (KMI), Nagoya University, Nagoya 464-8602, Japan

${ }^{b}$ Higgs Centre for Theoretical Physics, SUPA, School of Physics and Astronomy, University of Edinburgh, Edinburgh EH9 3JZ, UK

${ }^{c}$ Computing Research Center, High Energy Accelerator Research Organization (KEK), Tsukuba 305-0801, Japan

E-mail: ohki@kmi.nagoya-u.ac.jp

In search for a composite Higgs boson (techni-dilaton) in the walking technicolor, we present our preliminary results on the first observation of a light flavor-singlet scalar in a candidate theory for the walking technicolor, the $N_{f}=8 \mathrm{QCD}$, which was found in our previous paper to have spontaneous chiral symmetry breaking together with remnants of the conformality. Based on simulations with the HISQ-type action on several lattice sizes with various fermion masses, we find evidence of a flavor-singlet scalar meson with mass comparable to that of the Nambu-Goldstone pion in both the small fermion-mass region, where chiral perturbation theory works, and the intermediate fermion-mass region where the hyperscaling relation holds. We further discuss its chiral limit extrapolation in comparison with other states studied in our previous paper: the scalar has a mass much smaller than that of the vector meson, which is compared to the Nambu-Goldstone pion having a vanishing mass in that limit.

31st International Symposium on Lattice Field Theory - LATTICE 2013

July 29 - August 3, 2013

Mainz, Germany

* Speaker. 


\section{Introduction}

Recently a Higgs boson with mass around $125 \mathrm{GeV}$ was discovered at LHC. While it is consistent with the one in the Standard model (SM), there still exists a possibility that it is a composite particle in an underlying strongly coupled gauge theory. One such example is the techni-dilaton predicted as a naturally light fermionic bound state in the walking technicolor having approximate scale symmetry and a large anomalous dimension $\gamma_{m} \sim 1$ [1]. A composite Higgs as the technidilaton is a pseudo Nambu-Goldstone (NG) boson of the spontaneously broken approximate scale symmetry and is shown to be phenomenologically consistent with the current LHC data [2]. Thus the most urgent theoretical task to test the walking technicolor would be to check whether or not such a light flavor-singlet scalar bound state exists using first-principle calculations on the lattice. (For reviews on the lattice studies in search for candidates for the walking technicolor see [3].)

Actually, in a previous work [4], we observed a flavor-singlet fermionic scalar meson $(\sigma)$ lighter than the "pion" (corresponding to the NG pion in the broken phase) in the $N_{f}=12$ QCD, which was studied by us in another paper on the same setting [5] and was consistent with a conformal theory. Since the conformal theory should have no bound state ("unparticle") at the exact chiral limit, the light bound states are only possible in the presence of the fermion mass $m_{f}$ in such a way that it produces the confining forces (blowing-up coupling) in the infrared region below the fermion mass scale. A light scalar in such theory would not be regarded as a composite Higgs boson. Nevertheless the walking theory should have a similar light scalar bound state in a similar conformal dynamics, with the role of $m_{f}$ replaced by the dynamically generated mass of the fermion.

In this paper we indeed observe a light flavor-singlet scalar $\sigma$ in the $N_{f}=8 \mathrm{QCD}$, which was shown to be a good candidate for the walking technicolor in our previous work [6]. The $\sigma$ we observe could be a first evidence of a candidate for the composite Higgs as a techni-dilaton on the lattice. As in $N_{f}=12$ QCD [4], we extract the $m_{\sigma}$ from the correlation function of the $0^{++}$ fermion bilinear operator, which consists of both connected and (vacuum subtracted) disconnected contributions. We find that the $\sigma$ is as light as the NG pion similarly to $N_{f}=12$ QCD [4]. As suggested by our previous work [6] on other quantities, an approximate hyperscaling behavior is also expected for the $m_{\sigma}$ in the relatively heavier $m_{f}$ region, while near the chiral limit where the spontaneous chiral breaking effects become dominant, the $m_{\sigma}$ should be described by a polynomial function as a perturbation of $m_{f}$. We then discuss the chiral limit extrapolation of $m_{\sigma}$ in a way consistent with the chiral perturbation theory.

In the next section, we explain the simulation setup and the methods for the flavor-singlet scalar measurement. In Section 3, we show the results on the correlation functions, the $m_{\sigma}$ as a function of $m_{f}$. In Section 4, we summarize our results and discuss the implications of light scalar in the chiral limit for a composite Higgs scenario. All the results shown here are preliminary.

\section{Lattice setup}

The gauge configurations for $\mathrm{SU}(3)$ gauge theory with eight fundamental fermions are generated by the HMC algorithm with tree-level Symanzik gauge action and HISQ (highly improved staggered quark) action without tadpole improvement and mass correction in the Naik term. By 
using two degenerate staggered fermion species, we carry out simulations on three different lattice volumes $\left(V=L^{3}\right) L=18,24$, and 30 , with fixed aspect ratio $T / L=4 / 3$ at a single lattice spacing $\left(\beta \equiv 6 / g^{2}=3.8\right)$ for five different fermion masses $\left(m_{f}=0.02,0.04,0.06,0.08,0.10\right)$. For each parameter, we accumulate more than 5000 trajectories, and perform measurements every 2 trajectories. Such a number of configurations allows us to obtain a reasonable signal of $m_{\sigma}$. The statistical error is estimated by the standard jackknife method with bin size of more than 100 trajectories.

For the measurement of the flavor-singlet scalar, we use the following local staggered fermion bilinear operator

$$
O_{S}(t)=\sum_{i} \sum_{x} \bar{\chi}_{i}(x, t) \chi_{i}(x, t)
$$

where $i$ denotes the staggered fermion species, $i=1,2$. Using this operator we measure the twopoint correlation function $\left\langle O_{S}(t) O_{S}(0)\right\rangle \propto 2 D(t)-C(t)$, where $C(t)$ and $D(t)$ are the connected and the vacuum subtracted disconnected correlators, respectively. The factor 2 in front of $D(t)$ is due to the number of species. For the calculation of $D(t)$, which is essential to estimate the $\sigma$ correlator, we need to calculate the inverse of the Dirac operator for all the space-time points $(x, t)$. In order to reduce the computational cost of the inversion, we use the stochastic estimator with noise vectors for space-time and color. The large fluctuation coming from random noise can be efficiently reduced by employing the noise reduction technique for staggered fermions [7 8 , which was already applied in previous studies, for example, the calculation of the flavor-singlet pseudo-scalar [8, 9], the chiral condensate [10], and also the $\sigma$ in $N_{f}=12$ QCD [11 4]. The chosen number of noise vectors for each gauge configuration is 64 to sufficiently suppress the fluctuation of random noise compared to gauge fluctuation. We tested the calculation method in the $N_{f}=12$ QCD case as reported in Ref. [4].

In the staggered fermion formulation, the scalar operator in Eq. (2.1) overlaps not only with the $\sigma$, but also with the pseudo-scalar state $\left(\pi_{\overline{S C}}\right)$, which is the staggered parity partner of $\sigma$ and has the staggered spin-taste structure $\left(\gamma_{4} \gamma_{5} \otimes \xi_{4} \xi_{5}\right)$. In order to reduce the contribution from the parity partner we use the projection $C_{+}(t) \equiv 2 C(t)+C(t+1)+C(t-1)$ at even $t$. The full correlator $2 D_{+}(t)-C_{+}(t)$ in the large $t$ region behaves as

$$
2 D_{+}(t)-C_{+}(t)=A_{\sigma}(t)
$$

where $A_{H}(t)=A_{H}\left(e^{-m_{H} t}+e^{-m_{H}(T-t)}\right)$. The connected correlator $C_{+}(t)$ in the large $t$ region can be regarded as $A_{a_{0}}(t)$ where $a_{0}$ is the flavor non-singlet scalar state. Thus, the asymptotic behavior of $2 D_{+}(t)$ is given by

$$
2 D_{+}(t)=A_{\sigma}(t)+A_{a_{0}}(t) .
$$

This means that both $2 D_{+}(t)-C_{+}(t)$ and $2 D_{+}(t)$ can be used to extract $m_{\sigma}$ from their ground state masses, if $m_{\sigma}<m_{a_{0}}$. We will discuss this point later. Another projection $C_{-}(t) \equiv 2 C(t)-C(t+$ 1) $-C(t-1)$ at even $t$ is also used to obtain the $\pi_{S C}$ state, which is the parity partner of $a_{0}$.

\section{Result}

Figure 1 shows a typical result of $-C(t)$ and $2 D(t)$ for $L=30, m_{f}=0.02$. As shown in the figure, we can obtain a good signal for $2 D(t)$ thanks to a large statistics and the noise reduction 
technique. In the large $t$ region, $2 D(t)$ behaves as a smooth function of $t$. This result indicates that the taste symmetry breaking effects on the parity partner are small [4 thanks to utilizing the HISQ-type action. The smallness of the taste symmetry breaking was also observed in other meson masses in our previous work [6].

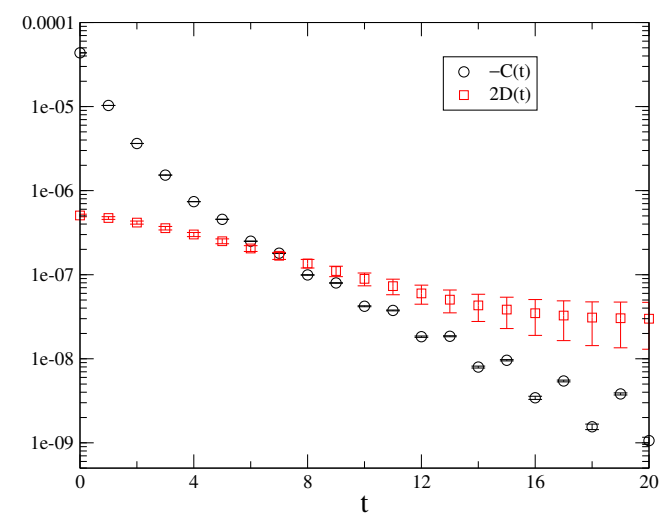

Figure 1: Connected $-C(t)$ and disconnected $2 D(t)$ correlators for $L=30, m_{f}=0.02$.

The left panel of Fig. 2] shows all the parity-projected correlators constructed from the $C(t)$ and $D(t)$ for $L=30, m_{f}=0.02$. We see that the full correlator $2 D_{+}(t)-C_{+}(t)$ at large $t$ is dominated by $2 D_{+}(t)$. Accordingly, the effective mass obtained from $2 D_{+}(t)$ becomes consistent with that obtained from $2 D_{+}(t)-C_{+}(t)$ as shown in the right panel of Fig. 2 This property allows us to evaluate the flavor-singlet scalar mass from the correlator $2 D_{+}(t)$. The advantage of using $2 D_{+}(t)$ is that the plateau appears at small $t$ owing to the cancellation between the $a_{0}$ and the contamination from excited states of the $\sigma$. The plateau of $2 D_{+}(t)$ enables us to determine the effective mass with relatively smaller statistical error. We fit $2 D_{+}(t)$ with a single cosh form in the range $t_{\min }=6$ and $t_{\max }=T / 2$ to obtain $m_{\sigma}$ for all the values of $m_{f}$. Comparing this with the rest of spectrum, we find that the $\sigma$ is not heavier than $\pi_{S C}$, whose effective mass corresponds to the one of $C_{-}(t)$ in the right panel of Fig. 2. As for the $a_{0}$ corresponding to $-C_{+}(t)$, the result would suggest $m_{a_{0}}>m_{\sigma}$, as we expected, although we do not obtain a good effective mass plateau in our lattice volume.
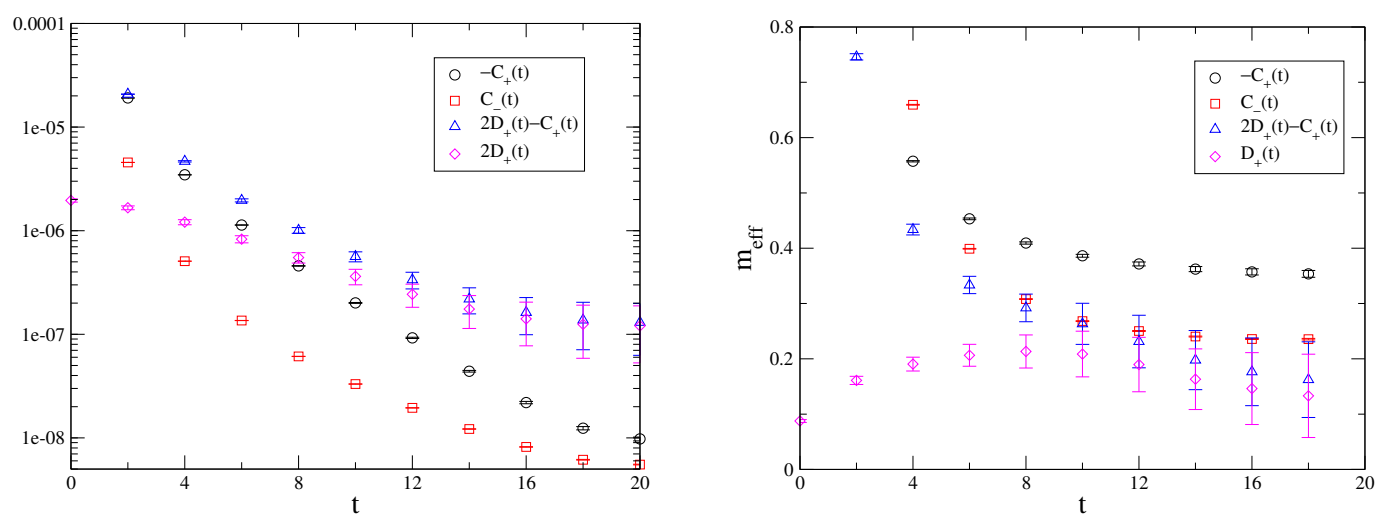

Figure 2: Parity-projected correlators for the different channels constructed from $C(t)$ and $D(t)$ (Left), and their effective masses (Right) for $L=30, m_{f}=0.02$.

The left panel of Fig. 3 presents fit results of the $m_{\sigma}$ as a function of $m_{f}$ for each volume, 

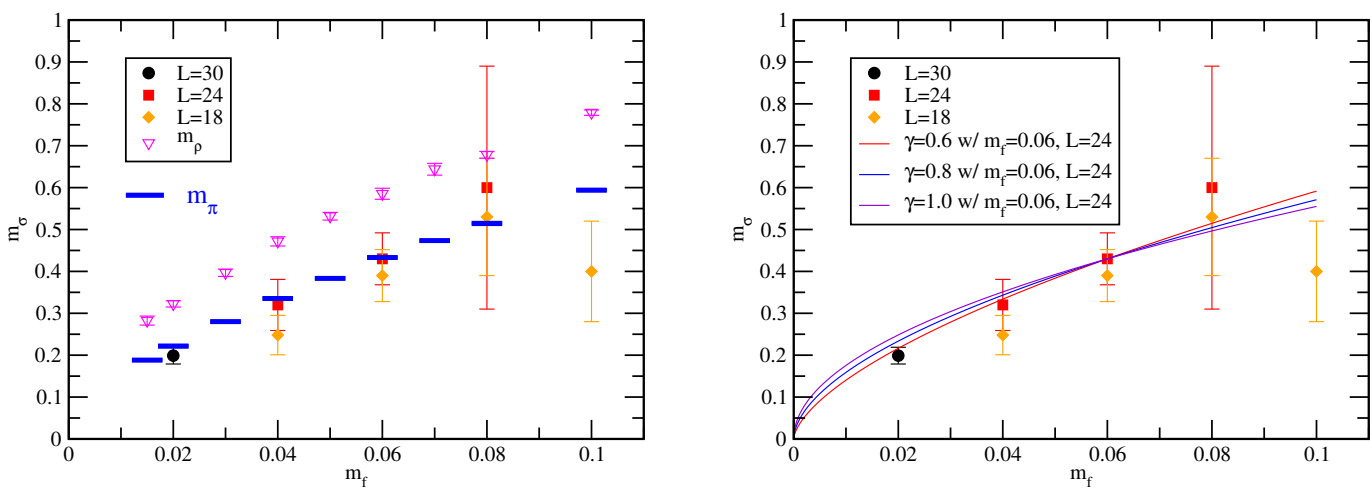

Figure 3: (Left) Flavor-singlet scalar mass $m_{\sigma}$ as a function of $m_{f}$ for each volume. The errors are only statistical. For comparison also plotted are $m_{\pi}$ and $m_{\rho}$ observed in Ref. [6]. (Right) Comparison of the $m_{\sigma}$ data with the hyperscaling curve(s) for the value(s) of $\gamma$ observed in Ref. [6] (see text), with the absolute value normalized to the data at $L=24, m_{f}=0.06$.

together with other masses $m_{\pi}$ and $m_{\rho}$ taken from Ref. [6]. The errors are only statistical. The two results on the different volumes $L=18$ and 24 at $m_{f}=0.04$ are consistent within the statistical error, so that we expect finite size effects to be small at heavier $m_{f}$. It is remarkable that all the $m_{\sigma}$ results at the measured $m_{f}$ are comparable to $m_{\pi}$. This feature is different from that of ordinary QCD with small $N_{f}$, and similar to the one observed in $N_{f}=12$ QCD [4]. It is also to be noted that $m_{\sigma}$ is much smaller than $m_{\rho}$.

Since we observed an approximate hyperscaling relation of other physical quantities for relatively heavy $m_{f}, m_{f} \geq 0.05$, interpreted as a remnant of conformality in our previous study [6], we also expect it for $\sigma$ in this region. We plot the hyperscaling curves $m_{\sigma}=C\left(m_{f}\right)^{1 /(1+\gamma)}$ in the right panel of Fig. 3, where $\gamma=0.6,0.8$ and 1.0 are values of $m_{\pi}, m_{\rho}$ and $F_{\pi}$, respectively, observed in Ref. [6], and the value of $C$ is matched to the $\sigma$ data at $m_{f}=0.06, L=24$. The data are roughly consistent with the hyperscaling relation with $\gamma=0.6-1.0$ within the errors.

On the other hand, the small $m_{f}$ region, $m_{f} \leq 0.04$, was shown to be consistent with the spontaneous chiral symmetry breaking [6]. We have seen in Fig. 3 that $\sigma$ is much lighter than $\rho$, and is as light as $\pi$ all the way down to the small $m_{f}$ region well described by the chiral perturbation theory, at least in the present data. This would imply a light composite scalar in the chiral limit in the walking theory.

\section{Summary and Discussion}

We have observed for the first time a light flavor-singlet scalar meson in the $N_{f}=8 \mathrm{QCD}$, which was shown to be a good candidate for the walking technicolor theory in our previous study. The observed mass is as light as the one of $\pi$ in the simulation parameters region. Measuring disconnected correlator was critical to the achievement. Our results are encouraging in search for the walking technicolor in view of the $125 \mathrm{GeV}$ Higgs at LHC.

Although our results are very preliminary and the statistical error is large, we discuss the chiral limit extrapolation of $m_{\sigma}$. As we observed in the previous paper [6], other physical quantities in the small $m_{f}$ region are described by the chiral perturbation theory fit. In Fig. 4 we plot the results 
of ChPT-like fits of $m_{\pi}$ and $m_{\rho}$ with the range $0.015 \leq m_{f} \leq 0.04$ (4 data) using the following fit functions [6],

$$
m_{\pi}^{2}=c_{1}^{\pi} m_{f}+c_{2}^{\pi} m_{f}^{2}, \quad m_{\rho}=c_{0}^{\rho}+c_{1}^{\rho} m_{f}+c_{2}^{\rho} m_{f}^{2} .
$$

From the data in this region, we try to estimate the $m_{\sigma}$ in the chiral limit. Note that $\sigma$ in the chiral limit can be a bound state only in the presence of fermion mass dynamically generated by the chiral symmetry breaking, which breaks scale symmetry explicitly as well as spontaneously. Hence its chiral limit mass should not be zero in the same way as $\rho$, so that the chiral fit should have the same functional form as that of $\rho$. We carry out the chiral extrapolation with just the lightest two points on $L \geq 24$ and hence use the linear fit,

$$
m_{\sigma}=c_{0}^{\sigma}+c_{1}^{\sigma} m_{f} .
$$

The fit result is shown in Fig. 4. The value in the chiral limit reads $m_{\sigma}=0.08(7)$. Then we have

$$
\frac{m_{\sigma}}{m_{\rho}}=0.5(5), \quad \frac{m_{\sigma}}{F_{\pi} / \sqrt{2}}=4(4),
$$

in the chiral limit $\left(F_{\pi} / \sqrt{2}\right.$ corresponds to $f_{\pi}=93 \mathrm{MeV}$ in the real-life QCD). Note that in a typical walking technicolor model, the one-family (four-weak-doublets) model with $N_{f}=8$, we have $F_{\pi} / \sqrt{2} \simeq 123 \mathrm{GeV}$. Within the error our results accommodate the $125 \mathrm{GeV}$ Higgs boson.

Here we note another possible signature of the walking behavior to be observed on the lattice data. As we mentioned above, when the chiral symmetry is spontaneously broken, the chiral limit of $m_{\sigma}$ should be non-zero due to the very presence of the dynamically generated mass of the fermion, while $m_{\pi}$ should go to zero as a NG boson for the same reason (non-zero dynamical mass). Hence, if $m_{\sigma}<m_{\pi}$ for larger $m_{f}$ as in our data, the chiral extrapolation of $m_{\sigma}$ and $m_{\pi}$ must be crossing to $m_{\sigma}>m_{\pi}$ at a certain smaller $m_{f}$. This never occurs in the conformal phase, since all the masses should obey the hyperscaling relations, and the ratio of $m_{\sigma} / m_{\pi}$ becomes a constant towards the chiral limit. Therefore, the observation of such a crossing phenomenon could be another signal of a walking theory. This will occur in much smaller $m_{f}$ region than in the present calculation, as seen from our rough chiral extrapolation in Fig. 4 In order to directly check the crossing on the lattice, we will need simulations at even smaller fermion masses and larger volumes.

Besides increasing statistics and obtaining more accurate $m_{\sigma}$ results in future, we shall construct a flavor-singlet scalar operator by gluonic operators (glueball) to check a consistency between the ground state masses extracted from different operators, as was studied in the $N_{f}=12$ QCD [4] 12]. We also would need to investigate lattice discretization effects in this theory.

\section{Acknowledgments}

Numerical calculations have been carried out on the high-performance computing system $\varphi$ at KMI, Nagoya University, and the computer facilities of the Research Institute for Information Technology in Kyushu University. This work is supported by the JSPS Grant-in-Aid for Scientific Research (S) No.22224003, (C) No.23540300 (K.Y.), for Young Scientists (B) No.25800139 (H.O.) and No.25800138 (T.Y.), and also by Grants-in-Aid of the Japanese Ministry for Scientific Research on Innovative Areas No.23105708 (T.Y.). E.R. was supported by a SUPA Prize Studentship and a FY2012 JSPS Postdoctoral Fellowship for Foreign Researchers (short-term). 


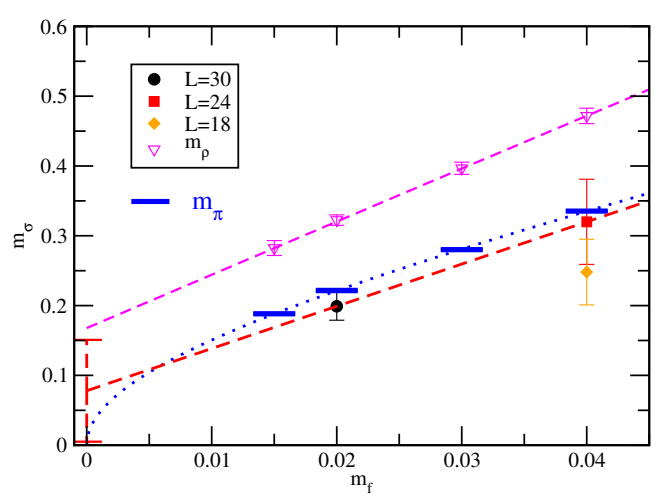

Figure 4: Fit result of the chiral extrapolation for $m_{\sigma}$. For comparison, other spectra of $m_{\pi}$ and $m_{\rho}$ and their chiral fits in Ref. [6] are also shown.

\section{References}

[1] K. Yamawaki, M. Bando and K. -i. Matumoto, Phys. Rev. Lett. 56, 1335 (1986).

[2] S. Matsuzaki and K. Yamawaki, Phys. Lett. B 719, 378 (2013); Phys. Rev. D 86, 115004 (2012); S. Matsuzaki, arXiv:1304.4882 [hep-ph].

[3] J. Kuti, PoS LATTICE 2013004 (2013); J. Giedt, PoS LATTICE 2012006 (2012); E. T. Neil, PoS LATTICE 2011, 009 (2011), and references therein.

[4] Y. Aoki, T. Aoyama, M. Kurachi, T. Maskawa, K. -i. Nagai, H. Ohki, E. Rinaldi, A. Shibata, K. Yamawaki and T. Yamazaki (LatKMI collaboration), arXiv:1305.6006 [hep-lat]; arXiv:1302.4577 [hep-lat].

[5] Y. Aoki, T. Aoyama, M. Kurachi, T. Maskawa, K. -i. Nagai, H. Ohki, A. Shibata, K. Yamawaki and T. Yamazaki (LatKMI collaboration), Phys. Rev. D 86 (2012) 054506.

[6] Y. Aoki, T. Aoyama, M. Kurachi, T. Maskawa, K. -i. Nagai, H. Ohki, A. Shibata, K. Yamawaki and T. Yamazaki (LatKMI collaboration), Phys. Rev. D 87 (2013) 094511, arXiv:1302.6859 [hep-lat].

[7] G. W. Kilcup and S. R. Sharpe, Nucl. Phys. B 283, 493 (1987).

[8] L. Venkataraman and G. Kilcup, [hep-lat/9711006].

[9] E. B. Gregory, A. C. Irving, C. M. Richards and C. McNeile, Phys. Rev. D 77, 065019 (2008).

[10] C. McNeile, A. Bazavov, C. T. H. Davies, R. J. Dowdall, K. Hornbostel, G. P. Lepage and H. D. Trottier, Phys. Rev. D 87, no. 3, 034503 (2013).

[11] X. -Y. Jin and R. D. Mawhinney, PoS LATTICE 2011, 066 (2011) [arXiv:1203.5855 [hep-lat]].

[12] Y. Aoki, T. Aoyama, M. Kurachi, T. Maskawa, K. Miura, K. -i. Nagai, H. Ohki, E. Rinaldi, A. Shibata, K. Yamawaki and T. Yamazaki (LatKMI collaboration), PoS LATTICE 2013, 073 (2013) 